\title{
Oscillations and hippocampal-prefrontal synchrony
}

\author{
Laura Lee Colgin \\ Center for Learning and Memory, Department of Neurobiology, The University of Texas at Austin, \\ 1 University Station Stop C7000, Austin, TX 78712, USA
}

\begin{abstract}
The hippocampus, a structure required for many types of memory, connects to the medial prefrontal cortex, an area that helps direct neuronal information streams during intentional behaviors. Increasing evidence suggests that oscillations regulate communication between these two regions. Theta rhythms may facilitate hippocampal inputs to the medial prefrontal cortex during mnemonic tasks and may also integrate series of functionally relevant gamma-mediated cell assemblies in the medial prefrontal cortex. During slow-wave sleep, temporal coordination of hippocampal sharp wave-ripples and medial prefrontal cortex spindles may be an important component of the process by which memories become hippocampus-independent. Studies using rodent models indicate that oscillatory phase-locking is disturbed in schizophrenia, emphasizing the need for more studies of oscillatory synchrony in the hippocampal-prefrontal network.
\end{abstract}

\section{Introduction}

The hippocampus is required for several types of memory [1]. It must cooperate with other structures that are involved in learning and memory, and brain rhythms are thought to be important for coordinating these interactions. When large groups of neurons synchronize their electrical activity in a periodic manner, brain rhythms (or oscillations) emerge in local field potential (LFP) recordings. The hippocampus exhibits three main classes of rhythms that are associated with particular behavioral states [2]: theta, gamma, and sharp waveripples. Theta rhythms $(\sim 5-10 \mathrm{~Hz})$ occur during active behaviors as well as REM sleep [3] and are believed to be important for learning and memory [4]. Gamma oscillations are faster waves $(\sim 25-140 \mathrm{~Hz})$ that occur during many behaviors but are largest when theta rhythms are present [5]. Ripples are very fast oscillations $(\sim 150-300 \mathrm{~Hz})$ that are superimposed on slow and irregularly occurring 'sharp waves' $(\sim 1-10 \mathrm{~Hz})$. Sharp-wave ripple complexes emerge during slow-wave sleep and periods of inactivity [6]. Each of these three classes of rhythms is believed to play a unique role in coordinating interactions between the hippocampus and the systems with which it communicates.

One region that interacts with the hippocampus is the medial prefrontal cortex (mPFC). The $\mathrm{mPFC}$ is important for working memory, as well as executive functions including decisionmaking, goal-oriented behaviors, and attentional selection of task-relevant information [7]. The mPFC receives direct projections from ventral CA1 and subiculum subfields of the hippocampus [8,9], and these synapses are plastic [10]. The hippocampus is believed to activate $\mathrm{mPFC}$ during behaviors in which functions of mPFC are required. For example, the hippocampus may encode a memory of a particular circumstance that is associated with a particular goal-directed behavior. When this circumstance arises later, the hippocampus may

(C) 2011 Elsevier Ltd. All rights reserved.

Corresponding author: Colgin, Laura Lee (colgin@mail.clm.utexas.edu). 
retrieve its memory of this circumstance and communicate to the prefrontal cortex so that behavior can be adjusted to achieve the desired goal.

I review recent studies that support the hypothesis that rhythms facilitate functional interactions between the hippocampus and $\mathrm{mPFC}$ during behaviors requiring both regions. Owing to the complexities related to defining homologous areas of the prefrontal cortex across species [9,11], this review focuses on rodent studies. However, coherent oscillations in the hippocampus and mPFC are believed to mediate memory operations in humans (e.g. $[12,13])$ and other species also.

\section{Theta interactions between MPFC and hippocampus}

Theta rhythms coordinate the activity of neurons on a relatively slow time scale. Slow oscillations are capable of coordinating activity across widespread networks of neurons because neurons in areas that are separated by long conduction delays can still be activated within the same oscillatory cycle [14]. Monosynaptic delays between the hippocampus and $\mathrm{mPFC}$ have been reported to be $\sim 15 \mathrm{~ms}$ [15]. Thus, the $\sim 150 \mathrm{~ms}$ period of a theta cycle would certainly be capable of coordinating direct activations between the two regions and plausibly could tolerate polysynaptic interactions as well. In line with this idea, several studies have recorded simultaneously from the hippocampus and mPFC and observed coherent theta rhythms $\left[16,17,18^{\circ \bullet}, 19^{\circ}\right]$, as well as correlations between spike times of mPFC neurons and hippocampal theta phase $\left(\left[16,18^{\bullet}, 19^{\bullet *}, 20-22,23^{\bullet}, 24,25^{\circ}\right]\right.$; Figure 1a).

The first study to demonstrate theta phase-locking between rat hippocampus and mPFC reported that approximately $40 \%$ of $\mathrm{mPFC}$ neurons were significantly phase-locked to CA 1 theta rhythms [20]. mPFC spikes were maximally phase-locked to CA1 theta rhythms that occurred $\sim 50 \mathrm{~ms}$ earlier, suggesting that CA1 theta entrains $\mathrm{mPFC}$ firing within the time scale of a single theta cycle. In this seminal study, recordings were collected during a variety of behaviors, leaving open the question of whether effects were behaviorally relevant. Shortly afterward, hippocampal theta phase-locking of mPFC neurons was reported to be associated with task-specific changes in $\mathrm{mPFC}$ firing rates in spatial navigation tasks [21]. Consistent with earlier findings [26], the firing rates of cells in mPFC correlated with particular aspects of behavior, such as running direction on a linear track. The majority of cells that exhibited theta phase-locking in only one running direction also had unidirectional behavioral correlates, and all cells that were theta phase-locked in both running directions also responded in both directions to particular behaviors. A subsequent study investigated the influence of hippocampal theta phase-locking of mPFC neurons on working memory, one of the main functions associated with mPFC [16]. Rats were tested on a continuous spatial alternation task that included choice epochs requiring working memory and forced turn epochs with no working memory requirements. mPFC cells were more strongly phaselocked to hippocampal theta during correct choice epochs than during forced turns and errors. Correct choices were also associated with enhanced theta coherence between mPFC and hippocampus. Similar findings were obtained in another study of memory in which rats performed a delayed non-match to sample task [24]. Nearly half of mPFC cells were phaselocked to hippocampal theta on correct trials, while less than $20 \%$ of cells were phaselocked on error trials. Taken together, the results of these studies support the conclusion that mPFC-hippocampal theta phase-locking is relevant for functions that are carried out by the hippocampus and $\mathrm{mPFC}$, such as goal directed behavior during navigation tasks and spatial working memory.

If theta phase-locking between hippocampus and $\mathrm{mPFC}$ is necessary for certain memory operations, as suggested by the studies described above, then the development of theta phase-locking between the regions should parallel the acquisition of learned behavior. A 
recent study addressed this hypothesis by simultaneously recording from ventral or intermediate $\mathrm{CA} 1$ and $\mathrm{mPFC}$ in rats that learned reward contingency rules on a Y-maze $\left[19^{\circ}\right]$. Theta coherence between mPFC and CA1 increased at the decision point of the maze, and this increase was significantly greater after animals had successfully learned the rule associated with reward. Moreover, a higher percentage of mPFC neurons fired together on the same phase of hippocampal theta after learning. This enhancement of correlated firing may enable mPFC to more effectively activate its outputs after learning has occurred. Remarkably, the authors observed similar effects (i.e. increased theta coherence and synchrony of $\mathrm{mPFC}$ neurons) when dopamine was applied in $\mathrm{mPFC}$, raising the possibility that learning-induced increases in theta synchrony are mediated by dopaminergic inputs to $\mathrm{mPFC}$ that signal expectation of reward [27].

Many of the studies discussed above involved simultaneous recordings from $\mathrm{mPFC}$ and dorsal CA1; however, only ventral CA1 projects directly to mPFC $[8,9]$. The ventral hippocampus has functions that differ from the dorsal hippocampus, including regulation of anxiety-related behaviors [28]. A recent study recorded in mPFC and ventral and dorsal CA1 in order to investigate whether interregional oscillatory phase-locking influences the effects of anxiety on behavior [18*0]. In anxiety-inducing environments, including an elevated plus maze, positive correlations were observed between $\mathrm{mPFC}$ and ventral, but not dorsal, hippocampal theta rhythms, and mPFC cells were strongly phase-locked to ventral hippocampal theta. Remarkably, theta power in $\mathrm{mPFC}$ and coherence between mPFC and ventral hippocampus decreased immediately before animals entered anxiety-inducing zones of the maze and increased when animals entered the 'safe' closed arms of the maze, even though there were no significant differences in running speed between these two behaviors. These findings suggest the intriguing hypothesis that theta phase-locking between the ventral hippocampus and $\mathrm{mPFC}$ inhibits anxiety-provoking behaviors.

The above-described findings raise the question of how the hippocampus imposes theta phase-locking on mPFC. Understanding the cellular mechanisms of theta phase-locking will provide clues about how theta phase-locking in $\mathrm{mPFC}$ affects downstream areas that mediate behavior. A recent study addressed the cellular mechanism question by recording from mPFC pyramidal cells and inter-neurons during hippocampal theta rhythms [23*0]. Pyramidal cells in $\mathrm{mPFC}$ preferentially fired at around the same theta phase as pyramidal cells in the ventral hippocampus, suggesting that ventral hippocampal pyramidal cells may directly activate mPFC pyramidal cells during theta states. Interestingly, mPFC basket cells, which target the somatic and proximal dendritic regions of pyramidal cells, preferentially discharged at this same portion of the theta cycle. This raises the possibility that feedforward inhibition of mPFC pyramidal cells regulates the selection of task-relevant inputs during theta. Specifically, feed-forward inhibition may ensure that only the most excitable $\mathrm{mPFC}$ cells can fire before the onset of potent, basket cell-mediated inhibition. Paired pulse facilitation of mPFC responses to ventral hippocampal activation $[10,15]$ would then ensure that the same population of cells is more strongly excited, and thus again selected, on the subsequent theta cycle.

\section{Gamma interactions between mPFC and hippocampus}

Gamma oscillations co-occur with theta rhythms in the hippocampus. However, few studies have addressed the question of whether synchronous gamma activity between the hippocampus and mPFC affects mnemonic processing. Although more work is needed, a number of recent results have provided clues about how gamma may coordinate interactions between the regions on a fast time scale. 
In the hippocampus, the amplitude of gamma oscillations is modulated by theta phase [5]. This may imply that gamma-mediated cell assemblies are integrated across space and time by theta rhythms [29]. A recent study showed that mPFC gamma power is modulated by hippocampal theta phase (Figure 1b), with localized gamma bursts transiently occurring at different mPFC locations [22]. Thus, spatially distributed gamma-mediated mPFC cell assemblies could be linked within the same theta cycle, while sequences of gamma-mediated cell assemblies could be integrated across successive theta cycles. The correlation between hippocampal theta phase and $\mathrm{mPFC}$ gamma amplitude has been replicated in more recent studies $[18,30]$.

In the hippocampus, separate slow and fast gamma oscillations have been observed to preferentially occur on significantly different phases of theta $\left[31^{*}\right]$, raising the question of whether hippocampal theta-modulated gamma in $\mathrm{MPFC}$ is slow or fast. One study reported hippocampal theta modulation of slow $(33-55 \mathrm{~Hz})$ gamma oscillations in mPFC [30]; however, the original study reported hippocampal theta modulation of primarily fast $(>100$ $\mathrm{Hz}$ ) gamma in mPFC [22]. It remains unclear why frequency differences were observed between the two studies, but it is possible that hippocampal theta modulates slow and fast gamma in $\mathrm{mPFC}$ at different times, reminiscent of how hippocampal theta modulates slow and fast gamma oscillations at different times in CA1 [31*0. In line with this idea, separate slow and fast gamma peaks are apparent in coherence spectra for paired mPFC-ventral hippocampus recordings from mice exploring a familiar arena ([18*0], Figure 2). Moreover, a comparison of mPFC-hippocampus coherence spectra from wild-type mice reveals a trend toward increased hippocampal-mPFC coherence in the slow, but not fast, gamma frequency range during the choice phase of a delayed non-match-to-sample spatial memory task ([25*], Figure 3). These findings support the hypothesis that slow and fast gamma oscillations are functionally distinct in the hippocampal-mPFC network, as has been suggested previously for the entorhinal-hippocampal network [31*0] and the ventral striatum [32].

What purpose might separate slow and fast gamma oscillations serve in the mPFChippocampal network? Fast gamma oscillations in the hippocampus are coherent with fast gamma oscillations in the medial entorhinal cortex [31*0], an area that transmits information to the hippocampus about the current environment $[33,34]$. It is possible that $\mathrm{mPFC}$ fast gamma oscillations are coherent with fast gamma in both the hippocampus and the entorhinal cortex. If so, coherent fast gamma oscillations in the entorhinal-hippocampalmPFC network could coordinate information flow across the three areas during processing of information related to the external environment. Considering that mPFC plays an important role in attentional selection [7], one possibility is that fast gamma oscillations selectively activate neurons that code information related to attended stimuli. On the contrary, slow gamma oscillations in CA1 are coherent with slow gamma in CA3 [31*0], an area that is required for memory retrieval $[35,36]$. Thus, coherent slow gamma oscillations between $\mathrm{mPFC}$ and the hippocampus could serve to coordinate interactions between the hippocampus and mPFC during operations such as memory retrieval and working memory, in which neuronal codes can be evoked or maintained internally after the represented information is no longer present in the external environment.

\section{Oscillatory interactions between $\mathrm{mPFC}$ and hippocampus during slow- wave sleep}

One theory of memory consolidation posits that certain memories initially depend on the hippocampus but then gradually are transferred to the neocortex [37], possibly during slowwave sleep. In slow-wave sleep, hippocampal neurons that were co-active during wakefulness reactivate together during sharp wave-ripples [38]. Similarly, mPFC neurons that fired together during waking reactivate together during subsequent slow-wave sleep 
[39]. Reactivation of mPFC neurons may be coordinated by synchronously occurring hippocampal sharp wave-ripples and mPFC spindles because correlations between hippocampal and $\mathrm{mPFC}$ firing during slow-wave sleep occur preferentially around the time of sharp wave-ripples (Figure $1 \mathrm{c},\left[40^{\circ}\right]$ ), and $\mathrm{mPFC}$ spindles tend to occur shortly after sharp wave-ripples are seen in the hippocampus [41].

Taken together, the above findings suggest that ripple-related reactivation of CA1 neurons and subsequent spindle-related reactivation of $\mathrm{mPFC}$ neurons may facilitate transfer of memories from the hippocampus to the mPFC during slow-wave sleep. The highly synchronous firing of CA1 neurons during sharp wave-ripples probably activates neurons in the main output of CA1, the subiculum. If CA1 firing during sharp wave-ripples activates neurons in the subiculum and mPFC at roughly the same time, then the subiculum and mPFC could in turn simultaneously activate common targets, such as the deep layers of the entorhinal cortex [42], potentially leading to associative long-term potentiation. If so, then mPFC inputs could eventually activate such targets on their own, and this could potentially explain why some initially hippocampal-dependent memories gradually become dependent on the mPFC $[43,44]$.

\section{Disrupted hippocampal-mPFC synchrony in rodent models of schizophrenia}

The prefrontal cortex is one of the main brain regions implicated in the neuropathology of schizophrenia [45]. Moreover, disturbances in functional connectivity between the hippocampus and prefrontal cortex have been reported in schizophrenic patients [46,47]. In light of this, two recent studies have used rodent models of schizophrenia to investigate how oscillatory phase-locking between the hippocampus and mPFC is affected by the disease.

The first study investigated mPFC-hippocampal synchrony in the $D f(16) A^{+/}$strain of transgenic mice, which models a human chromosomal abnormality that is associated with a $\sim 30$ fold greater risk of developing schizophrenia $\left[25^{\circ}\right]$. Compared to wild-type mice, the mutant mice exhibited significantly reduced hippocampal theta phase-locking of mPFC neurons and decreased theta coherence between the hippocampus and mPFC. The mutants also showed significant deficits on a delayed non-match-to-sample spatial working memory task that correlated with reductions in hippocampal-prefrontal theta coherence. These results raise the possibility that deficient theta phase-locking between the regions contributes to working memory deficits associated with schizophrenia.

In the second study, pregnant rat dams were injected with a cytokine inducer to activate the maternal immune response during the prenatal period $\left[48^{\circ}\right]$ because epidemiological studies have linked maternal immune activation (MIA) during pregnancy to an increased risk of schizophrenia in offspring [49]. Coherence between the hippocampus and prefrontal cortex was significantly reduced in the delta $(2-4 \mathrm{~Hz})$, theta $(4-12 \mathrm{~Hz})$, beta $(12-30 \mathrm{~Hz})$, and low frequency gamma $(30-48 \mathrm{~Hz})$ bands in rats that were prenatally exposed to MIA. In accordance, $\mathrm{mPFC}$ neurons in MIA rats were significantly less phase-locked to hippocampal theta and gamma oscillations. Additionally, reductions in low frequency gamma mPFChippocampal coherence were correlated with deficits in prepulse inhibition to startle, a widely used measure of sensorimotor gating that is impaired in schizophrenics. These findings suggest that deficient oscillatory synchrony between the hippocampus and prefrontal cortex may contribute to the sensory gating deficits associated with schizophrenia. 


\section{Conclusions}

The above-discussed studies have advanced our knowledge of how oscillations affect functions that are handled jointly by the mPFC and hippocampus. Coherent theta oscillations coordinate interactions between the hippocampus and $\mathrm{mPFC}$ during complex cognitive operations such as working memory. Hippocampal theta modulation of mPFC gamma oscillations may also play an important role in coordinating interactions between the regions. Reactivation of mPFC neurons by hippocampal sharp wave-ripples may help to gradually transfer some memories from the hippocampus to the $\mathrm{mPFC}$ during slow-wave sleep. Disturbances in oscillatory phase-locking between the hippocampus and mPFC may contribute to cognitive deficits in schizophrenia. Uncovering oscillatory mechanisms in the $\mathrm{mPFC}$ and hippocampus is thus very important (Table 1). Future results in this area may pave the way toward development of novel treatments for schizophrenia that aim to alleviate cognitive deficits by restoring normal patterns of oscillatory phase-locking between the hippocampus and mPFC.

\section{Acknowledgments}

I thank Michael Drew and Michael Mauk for helpful comments. Funding was provided by University of Texas start-up funds and grant P30 MH089900 from NIMH.

\section{References and recommended reading}

Papers of particular interest, published within the period of review, have been highlighted as:

- of special interest

•• of outstanding interest

1. Squire LR, Stark CE, Clark RE. The medial temporal lobe. Annu Rev Neurosci. 2004; 27:279-306. [PubMed: 15217334]

2. Buzsaki G, Leung LW, Vanderwolf CH. Cellular bases of hippocampal EEG in the behaving rat. Brain Res. 1983; 287:139-171. [PubMed: 6357356]

3. Vanderwolf $\mathrm{CH}$. Hippocampal electrical activity and voluntary movement in the rat. Electroencephalogr Clin Neurophysiol. 1969; 26 :407-418. [PubMed: 4183562]

4. Buzsaki G. Theta rhythm of navigation: link between path integration and landmark navigation, episodic and semantic memory. Hippocampus. 2005; 15:827-840. [PubMed: 16149082]

5. Bragin A, Jando G, Nadasdy Z, Hetke J, Wise K, Buzsaki G. Gamma (40-100 Hz) oscillation in the hippocampus of the behaving rat. J Neurosci. 1995; 15(1 Pt 1):47-60. [PubMed: 7823151]

6. Buzsaki G. Hippocampal sharp waves: their origin and significance. Brain Res. 1986; 398:242-252. [PubMed: 3026567]

7. Miller EK, Cohen JD. An integrative theory of prefrontal cortex function. Annu Rev Neurosci. 2001; 24:167-202. [PubMed: 11283309]

8. Jay TM, Witter MP. Distribution of hippocampal CA1 and subicular efferents in the prefrontal cortex of the rat studied by means of anterograde transport of Phaseolus vulgaris-leucoagglutinin. $\mathrm{J}$ Comp Neurol. 1991; 313:574-586. [PubMed: 1783682]

9. Hoover WB, Vertes RP. Anatomical analysis of afferent projections to the medial prefrontal cortex in the rat. Brain Struct Funct. 2007; 212:149-179. [PubMed: 17717690]

10. Laroche S, Jay TM, Thierry AM. Long-term potentiation in the prefrontal cortex following stimulation of the hippocampal CA1/subicular region. Neurosci Lett. 1990; 114:184-190. [PubMed: 2395531]

11. Nieuwenhuis IL, Takashima A. The role of the ventromedial prefrontal cortex in memory consolidation. Behav Brain Res. 2011; 218:325-334. [PubMed: 21147169] 
12. Anderson KL, Rajagovindan R, Ghacibeh GA, Meador KJ, Ding M. Theta oscillations mediate interaction between prefrontal cortex and medial temporal lobe in human memory. Cereb Cortex. 2010; 20:1604-1612. [PubMed: 19861635]

13. Sederberg PB, Schulze-Bonhage A, Madsen JR, Bromfield EB, Litt B, Brandt A, Kahana MJ. Gamma oscillations distinguish true from false memories. Psychol Sci. 2007; 18:927-932. [PubMed: 17958703]

14. Buzsaki G, Draguhn A. Neuronal oscillations in cortical networks. Science. 2004; 304:1926-1929. [PubMed: 15218136]

15. Jay TM, Thierry AM, Wiklund L, Glowinski J. Excitatory amino acid pathway from the hippocampus to the prefrontal cortex. Contribution of AMPA receptors in hippocampo-prefrontal cortex transmission. Eur J Neurosci. 1992; 4:1285-1295. [PubMed: 12106392]

16. Jones MW, Wilson MA. Theta rhythms coordinate hippocampal-prefrontal interactions in a spatial memory task. PLoS Biol. 2005; 3:e402. [PubMed: 16279838]

17. Young CK, McNaughton N. Coupling of theta oscillations between anterior and posterior midline cortex and with the hippocampus in freely behaving rats. Cereb Cortex. 2009; 19:24-40. [PubMed: 18453538]

18••. Adhikari A, Topiwala MA, Gordon JA. Synchronized activity between the ventral hippocampus and the medial prefrontal cortex during anxiety. Neuron. 2010; 65:257-269. In this study, the role of theta oscillatory phase-locking between mPFC and ventral hippocampus during anxietyrelated behaviors was investigated. Theta power between the ventral hippocampus and $\mathrm{mPFC}$ was more strongly correlated in anxiety-inducing environments than in familiar environments, and $\mathrm{mPFC}$ neurons showed enhanced phase-locking to $\mathrm{mPFC}$ and ventral hippocampal theta in anxiety-inducing environments. Theta power in $\mathrm{mPFC}$ and theta coherence between the hippocampus and mPFC decreased immediately before animals entered anxiety-inducing zones of an elevated plus maze. mPFC theta power and ventral hippocampal-mPFC theta coherence increased as animals entered 'safe' closed arms of the elevated plus maze. These effects could not be attributed to changes in running speed during the transitions between closed and open zones of the maze. The effects suggest that enhanced theta phase-locking in mPFC may help inhibit exploratory behavior during anxiety and also promote anxiolytic behaviors. [PubMed: 20152131]

19••. Benchenane K, Peyrache A, Khamassi M, Tierney PL, Gioanni Y, Battaglia FP, Wiener SI. Coherent theta oscillations and reorganization of spike timing in the hippocampal-prefrontal network upon learning. Neuron. 2010; 66:921-936. This study showed that theta coherence between $\mathrm{mPFC}$ and the hippocampus increases at the choice point of a Y-maze, particularly after rule acquisition. During elevated hippocampal-mPFC coherence and after learning, mPFC cells shifted their hippocampal theta phase preference, such that all mPFC pyramidal cells tended to fire synchronously. Similar effects (i.e. increased theta coherence and enhanced theta synchronized firing in $\mathrm{mPFC}$ ) were observed after dopamine application in $\mathrm{mPFC}$. Also, mPFC neurons that were co-active during periods of heightened $\mathrm{mPFC}$-hippocampal theta coherence were reactivated after hippocampal sharp wave-ripples only after learning had occurred. mPFC neurons that were co-active during periods of low theta coherence did not exhibit hippocampal sharp wave-triggered reactivation. The results support the hypothesis that theta phase-locking during waking behaviors strengthens connections between the hippocampus and $\mathrm{mPFC}$ that are subsequently reactivated during slow-wave sleep. [PubMed: 20620877]

20. Siapas AG, Lubenov EV, Wilson MA. Prefrontal phase locking to hippocampal theta oscillations. Neuron. 2005; 46:141-151. [PubMed: 15820700]

21. Hyman JM, Zilli EA, Paley AM, Hasselmo ME. Medial prefrontal cortex cells show dynamic modulation with the hippocampal theta rhythm dependent on behavior. Hippocampus. 2005; 15:739-749. [PubMed: 16015622]

22. Sirota A, Montgomery S, Fujisawa S, Isomura Y, Zugaro M, Buzsaki G. Entrainment of neocortical neurons and gamma oscillations by the hippocampal theta rhythm. Neuron. 2008; 60:683-697. [PubMed: 19038224]

23••. Hartwich K, Pollak T, Klausberger T. Distinct firing patterns of identified basket and dendritetargeting interneurons in the prefrontal cortex during hippocampal theta and local spindle oscillations. J Neurosci. 2009; 29:9563-9574. In this study, juxtacellularly labeled pyramidal 
cells and two classes of interneurons in mPFC were recorded simultaneously with CA1 theta rhythms and local spindle oscillations. Both pyramidal cells and basket cell interneurons in mPFC tended to fire phase-locked to hippocampal theta rhythms, with both types of cells exhibiting similar theta phase preferences. These two cell classes also tended to fire phase-locked to local spindle oscillations, with mPFC pyramidal cells preferentially firing on the trough and basket cells preferentially firing on the early ascending phase. Dendrite-targeting interneurons tended to stop firing during hippocampal theta rhythms and were not modulated by local spindle phase. The findings raise the possibility that feed-forward inhibition of mPFC pyramidal cells by $\mathrm{mPFC}$ basket cells plays a role in $\mathrm{mPFC}$ cell assembly selection during hippocampal theta. [PubMed: 19641119]

24. Hyman JM, Zilli EA, Paley AM, Hasselmo ME. Working memory performance correlates with prefrontal-hippocampal theta interactions but not with prefrontal neuron firing rates. Front Integr Neurosci. 2010; 4:1-13. [PubMed: 20161992]

25•. Sigurdsson T, Stark KL, Karayiorgou M, Gogos JA, Gordon JA. Impaired hippocampal-prefrontal synchrony in a genetic mouse model of schizophrenia. Nature. 2010; 464:763-767. This study showed that phase-locking of mPFC neurons to hippocampal theta during a working memory task was stronger in wild-type mice than in a transgenic mouse model of schizophrenia. Mutant mice took longer to learn the task, and the amount of mPFC-hippocampal theta coherence before training correlated negatively with the number of days that it took mutant mice to reach criterion performance. [PubMed: 20360742]

26. Jung MW, Qin Y, McNaughton BL, Barnes CA. Firing characteristics of deep layer neurons in prefrontal cortex in rats performing spatial working memory tasks. Cereb Cortex. 1998; 8:437450. [PubMed: 9722087]

27. Schultz W. Predictive reward signal of dopamine neurons. J Neurophysiol. 1998; 80:1-27. [PubMed: 9658025]

28. Kjelstrup KG, Tuvnes FA, Steffenach HA, Murison R, Moser EI, Moser MB. Reduced fear expression after lesions of the ventral hippocampus. Proc Natl Acad Sci USA. 2002; 99:1082510830. [PubMed: 12149439]

29. Jensen O, Colgin LL. Cross-frequency coupling between neuronal oscillations. Trends Cogn Sci. 2007; 11:267-269. [PubMed: 17548233]

30. Dzirasa K, Ramsey AJ, Takahashi DY, Stapleton J, Potes JM, Williams JK, Gainetdinov RR, Sameshima K, Caron MG, Nicolelis MA. Hyperdopaminergia and NMDA receptor hypofunction disrupt neural phase signaling. J Neurosci. 2009; 29:8215-8224. [PubMed: 19553461]

31••. Colgin LL, Denninger T, Fyhn M, Hafting T, Bonnevie T, Jensen O, Moser MB, Moser EI. Frequency of gamma oscillations routes flow of information in the hippocampus. Nature. 2009; 462:353-357. This paper provides evidence that separate slow and fast variants of gamma oscillations differentially route information flow in the entorhinal-hippocampal network. Coherent fast gamma oscillations were observed between CA1 and the medial entorhinal cortex, and coherent slow gamma oscillations occurred between CA1 and CA3. Medial entorhinal neurons were significantly phase-locked to fast, but not slow, gamma in CA1. By contrast, a significantly higher proportion of CA3 neurons were phase-locked to CA1 slow gamma than to CA1 fast gamma. In CA1, slow and fast gamma oscillations were maximal at significantly different phases of the underlying theta rhythms. The findings suggest that distinct frequencies of gamma oscillations in CA1 might help prevent interference between current spatial information arriving from the medial entorhinal cortex and memory retrieval-related information arriving from CA3. [PubMed: 19924214]

32. van der Meer MA, Redish AD. Low and high gamma oscillations in rat ventral striatum have distinct relationships to behavior, reward, and spiking activity on a learned spatial decision task. Front Integr Neurosci. 2009; 3:1-19. [PubMed: 19225578]

33. Hafting T, Fyhn M, Molden S, Moser MB, Moser EI. Microstructure of a spatial map in the entorhinal cortex. Nature. 2005; 436:801-806. [PubMed: 15965463]

34. Fyhn M, Hafting T, Treves A, Moser MB, Moser EI. Hippocampal remapping and grid realignment in entorhinal cortex. Nature. 2007; 446:190-194. [PubMed: 17322902] 
35. Sutherland RJ, Whishaw IQ, Kolb B. A behavioural analysis of spatial localization following electrolytic, kainate- or colchicine-induced damage to the hippocampal formation in the rat. Behav Brain Res. 1983; 7:133-153. [PubMed: 6830648]

36. Steffenach HA, Sloviter RS, Moser EI, Moser MB. Impaired retention of spatial memory after transection of longitudinally oriented axons of hippocampal CA3 pyramidal cells. Proc Natl Acad Sci USA. 2002; 99:3194-3198. [PubMed: 11867718]

37. Squire LR, Alvarez P. Retrograde amnesia and memory consolidation: a neurobiological perspective. Curr Opin Neurobiol. 1995; 5:169-177. [PubMed: 7620304]

38. Wilson MA, McNaughton BL. Reactivation of hippocampal ensemble memories during sleep. Science. 1994; 265:676-679. [PubMed: 8036517]

39. Euston DR, Tatsuno M, McNaughton BL. Fast-forward playback of recent memory sequences in prefrontal cortex during sleep. Science. 2007; 318:1147-1150. [PubMed: 18006749]

40•. Wierzynski CM, Lubenov EV, Gu M, Siapas AG. State-dependent spike-timing relationships between hippocampal and prefrontal circuits during sleep. Neuron. 2009; 61:587-596. In this study, simultaneous $\mathrm{mPFC}$ and CA1 recordings were collected during slow-wave sleep. Approximately $10 \%$ of hippocampal-prefrontal cell pairs were significantly correlated during slow-wave sleep. In the majority of correlated cell pairs, prefrontal cells preferentially fired a few tens of milliseconds after CA1 cells. Correlated mPFC-hippocampal cell activity was found to primarily occur around the time of hippocampal sharp wave-ripples. Closer examination revealed that sharp wave-ripples that were associated with low levels of CA1 bursting did not activate mPFC cells or spindles. However, sharp wave-ripples that were associated with strong CA1 bursting promoted bursting in mPFC that emerged $\sim 30 \mathrm{~ms}$ after CA1 bursting and triggered a second response in $\mathrm{mPFC}$ that emerged $\sim 100 \mathrm{~ms}$ later. The amplitude of mPFC spindles was also enhanced following sharp wave-associated bursting in CA1. The findings suggest that particularly strong sharp wave-ripples may trigger strong, reverberating responses in the $\mathrm{mPFC}$ network. [PubMed: 19249278]

41. Siapas AG, Wilson MA. Coordinated interactions between hippocampal ripples and cortical spindles during slow-wave sleep. Neuron. 1998; 21:1123-1128. [PubMed: 9856467]

42. Witter, MP.; Amaral, DG. Hippocampal formation. In: Paxinos, G., editor. The Rat Nervous System. San Diego, CA: Elsevier Academic Press; 2004.

43. Takehara K, Kawahara S, Kirino Y. Time-dependent reorganization of the brain components underlying memory retention in trace eyeblink conditioning. J Neurosci. 2003; 23:9897-9905. [PubMed: 14586019]

44. Maviel T, Durkin TP, Menzaghi F, Bontempi B. Sites of neocortical reorganization critical for remote spatial memory. Science. 2004; 305:96-99. [PubMed: 15232109]

45. Lewis DA, Hashimoto T, Volk DW. Cortical inhibitory neurons and schizophrenia. Nat Rev Neurosci. 2005; 6:312-324. [PubMed: 15803162]

46. Uhlhaas PJ, Singer W. Abnormal neural oscillations and synchrony in schizophrenia. Nat Rev Neurosci. 2010; 11:100-113. [PubMed: 20087360]

47. Woo TU, Spencer K, McCarley RW. Gamma oscillation deficits and the onset and early progression of schizophrenia. Harv Rev Psychiatry. 2010; 18:173-189. [PubMed: 20415633]

48. Dickerson DD, Wolff AR, Bilkey DK. Abnormal long-range neural synchrony in a maternal immune activation animal model of schizophrenia. J Neurosci. 2010; 30:12424-12431. This study utilized a rat model of schizophrenia that recreates maternal immune activation (MIA) in the prenatal period, a factor that correlates with an increased likelihood of schizophrenic offspring. MIA rats exhibited reduced mPFC-hippocampal coherence in the delta, theta, beta, and slow (but not fast) gamma frequency bands. No significant differences in oscillatory power were observed in hippocampus or mPFC between MIA and control rats. When MIA and control rats were pooled, a positive linear relationship was observed between hippocampal-mPFC slow $(\sim 30-48 \mathrm{~Hz})$ gamma coherence and the amount of paired pulse inhibition, a measure of sensory gating that is impaired in schizophrenics. mPFC neurons in MIA rats were significantly less phase-locked to theta and gamma oscillations in the hippocampus. The distribution of hippocampal gamma phases associated with mPFC spike times was bimodal in control animals and unimodal in MIA animals. This raises the possibility that normally there are two populations of mPFC neurons that prefer to fire at different gamma phases and that gamma coordination is 
selectively disrupted in one of these neuronal subtypes during schizophrenia. [PubMed: 20844137]

49. Patterson PH. Maternal infection: window on neuroimmune interactions in fetal brain development and mental illness. Curr Opin Neurobiol. 2002; 12:115-118. [PubMed: 11861174]

50••. Muzzio IA, Levita L, Kulkarni J, Monaco J, Kentros C, Stead M, Abbott LF, Kandel ER. Attention enhances the retrieval and stability of visuospatial and olfactory representations in the dorsal hippocampus. PLoS Biol. 2009; 7:1-20. In this study, mice were trained on one of two goal-oriented tasks. In the visuospatial version, reward was consistently delivered in the same location, but the scent of the bedding that covered the reward changed across trials, thus requiring animals to learn to attend to unchanging visuospatial cues and disregard olfactory cues. In the olfactory version, the reward was placed in different locations across trials, but the scent of the bedding that covered it remained the same, requiring animals to selectively attend to odors in order to locate the reward. The authors observed stable place fields in CA1 in the visuospatial version, but CA1 place field stability was impaired in animals that were required to attend to olfactory cues. In animals trained in the visuospatial task, CA1 place cell spikes were found to be phase-locked to slow gamma oscillations $(20-60 \mathrm{~Hz})$. Both slow gamma phase-locking of CA1 place cells and place field stability increased as animals learned to successfully perform the visuospatial task. The findings suggest that slow gamma phase-locking may play a role in retrieval of stable place cell representations.

$51 \bullet$. Keleman E, Fenton AA. Dynamic grouping of hippocampal neural activity during cognitive control of two spatial frames. PLoS Biol. 2010; 8:1-14. In this study, rats were trained in a twoframe, active place avoidance task in which they learned to avoid two shock zones: one that was defined by stationary visual cues in the room ('room frame') and one that was defined by olfactory cues and visual features on a rotating arena ('arena frame'). The authors first showed that correct performance on this task requires a fully intact hippocampal network. They found that $\sim 75 \%$ of CA1 place cells represented locations according to one frame and not the other, with about equal percentages of place cells coding each of the two frames. Cells that were concurrently active tended to code the same spatial frame. Across time, activation of cell assemblies that represented the room frame alternated with cell assemblies that coded the arena frame. Cell pairs that coded the same spatial frame discharged together preferentially within $\sim 25$ $\mathrm{ms}$ of each other. Considering that $25 \mathrm{~ms}$ corresponds to the period of a slow gamma $(\sim 40 \mathrm{~Hz})$ cycle, the results raise the possibility that slow gamma oscillations correlate the activity of CA1 neurons that code related information. The methodology described in this paper provides an ideal experimental paradigm for testing whether gamma phase-locking promotes transfer of taskrelevant hippocampal inputs to $\mathrm{mPFC}$, and whether this process plays a role in preventing interference between different streams of information. 


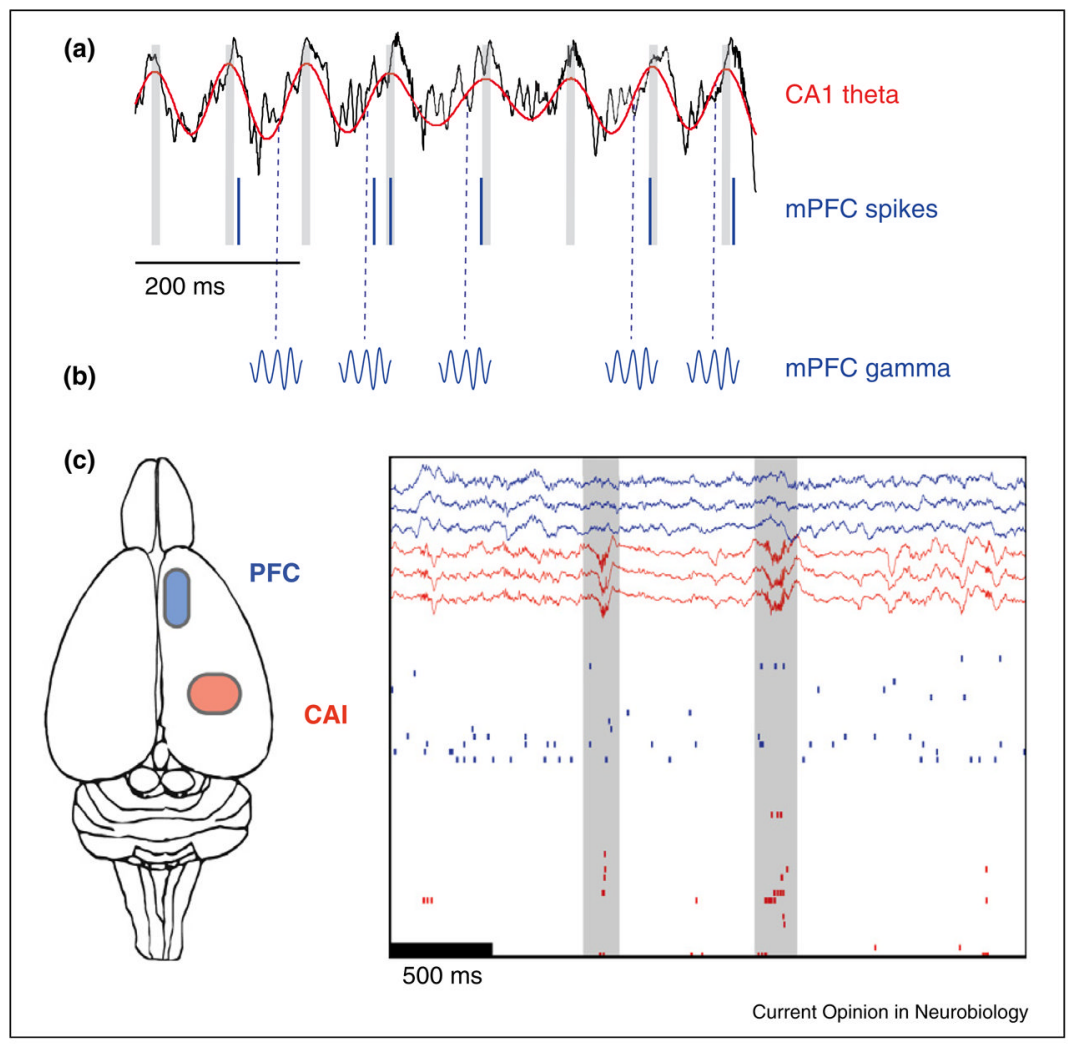

Figure 1.

Hippocampal theta and sharp wave-ripples coordinate neuronal activity in mPFC. (a) mPFC spikes are modulated by CA1 theta phase. An example field potential recording from CA1 is shown in black; the corresponding theta filtered recording is shown superimposed in red. Spikes recorded simultaneously from a mPFC neuron are shown below (vertical blue lines). Modified and reproduced from [25], with permission from Nature Publishing Group, Macmillan Publishers Ltd (http://www.nature.com). (b) Schematic illustrating the modulation of mPFC gamma amplitude by hippocampal theta phase $\left[18^{* *}, 22,30\right]$. Note that this effect was not measured in the study from which the theta recordings in (a) were obtained [ $\left.25^{\circ}\right]$; thus, hippocampal theta phase values (dashed vertical blue lines) depicted here for illustration of the general effect may not be accurate. (c) Simultaneous mPFC (blue) and CA1 (red) LFP recordings during slow-wave sleep are depicted above corresponding single unit recordings. Note that when sharp wave-ripples are apparent in the CA1 LFP recordings, synchronous bursts of $\mathrm{mPFC}$ and CA1 single unit activity can also be seen. Modified and reproduced from [40], with permission from Elsevier (http:// www.sciencedirect.com/science/journal/08966273). 


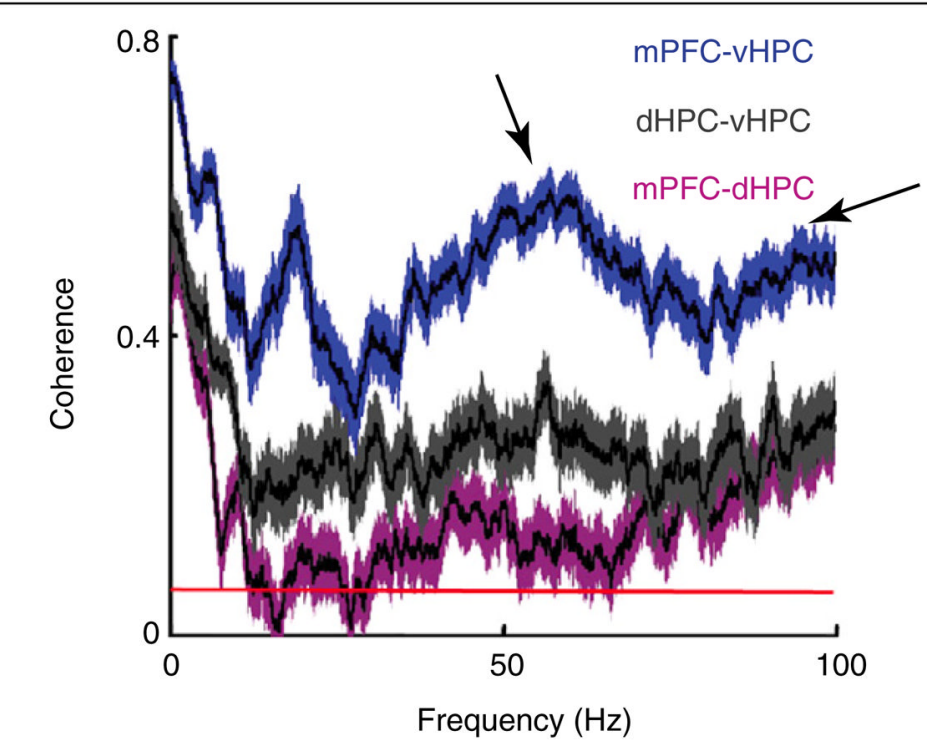

Current Opinion in Neurobiology

Figure 2.

Average coherence spectra for simultaneous LFP recordings from $\mathrm{mPFC}$ and ventral hippocampus (blue), dorsal and ventral hippocampi (gray), and $\mathrm{mPFC}$ and dorsal hippocampus (purple) in wild-type mice running between 7 and $15 \mathrm{~cm} / \mathrm{s}$ in a familiar arena. Shaded areas indicate $95 \%$ confidence intervals, and the red line at the bottom indicates the level of coherence expected by chance $(p<0.05)$. Note that two peaks can be seen in the gamma range, at $\sim 50$ and $100 \mathrm{~Hz}$ (indicated by arrows), in the coherence spectrum for simultaneous mPFC-ventral hippocampal recordings. Modified and reproduced from [18 ${ }^{\bullet}$ ], with permission from Elsevier (http://www.sciencedirect.com/science/journal/08966273). 


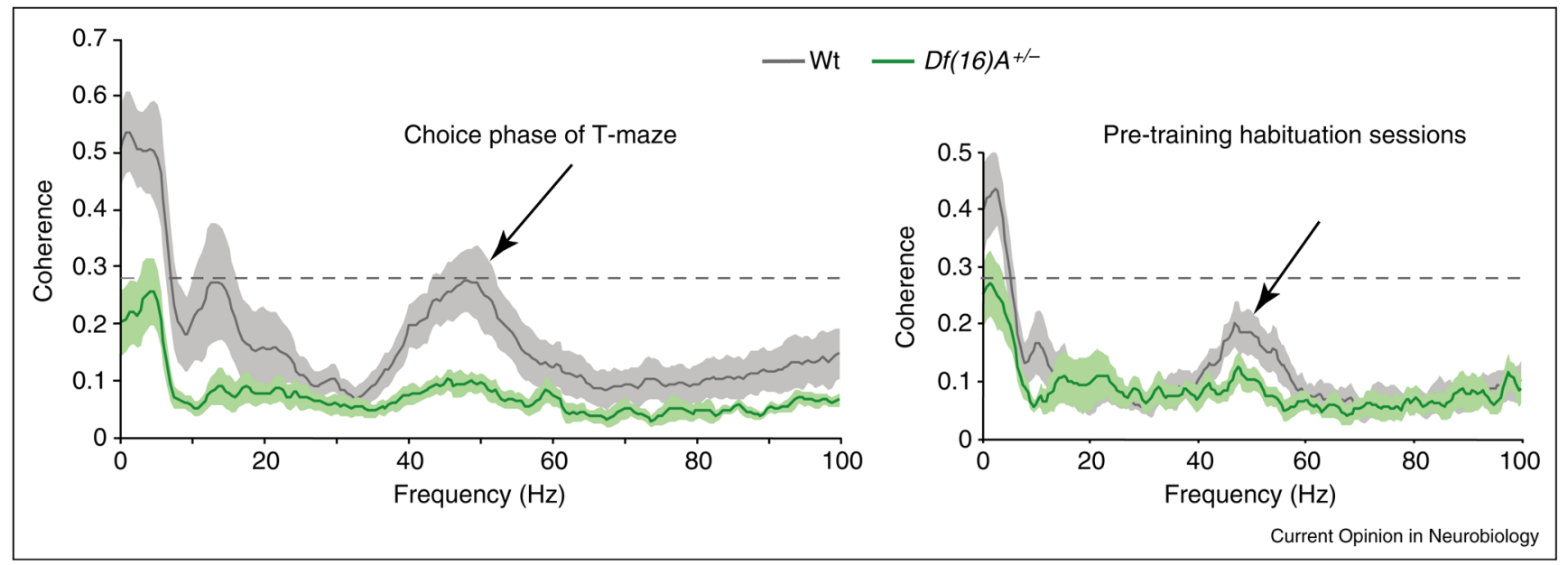

Figure 3.

Average coherence spectra for simultaneous LFP recordings from $\mathrm{mPFC}$ and CA1 in wildtype (gray) and $D f(16) A^{+/}$(green) mice. Shaded areas indicate the standard error of the mean. Coherence during the choice phase of a working memory task is shown on the left, while coherence during habituation sessions is shown on the right. CA1-mPFC coherence was reduced in mutant mice. Note that theta $(\sim 5 \mathrm{~Hz})$ and slow sgamma $(\sim 45 \mathrm{~Hz})$ coherence tended to be higher in wild-type mice when working memory processes were engaged. Modified and reproduced from [25 $5^{\circ}$, with permission from Nature Publishing Group, Macmillan Publishers Ltd (http://www.nature.com). 
Table 1

Outstanding issues

Does oscillatory synchrony across the mPFC-hippocampal network affects tasks that require animals to select relevant information and suppress irrelevant information (e.g. $\left.\left[50^{* *}, 51^{*}\right]\right)$ ?

Is the mPFC-hippocampal network phase-locked to the amygdala during fear-related behaviors?

Is the mPFC-hippocampal network phase-locked to the ventral striatum during reward-related tasks?

How can disturbances in oscillatory phase-locking between the hippocampus and MPFC be counteracted in schizophrenia? 